\title{
NOTE ON THE TRANSLATION
}

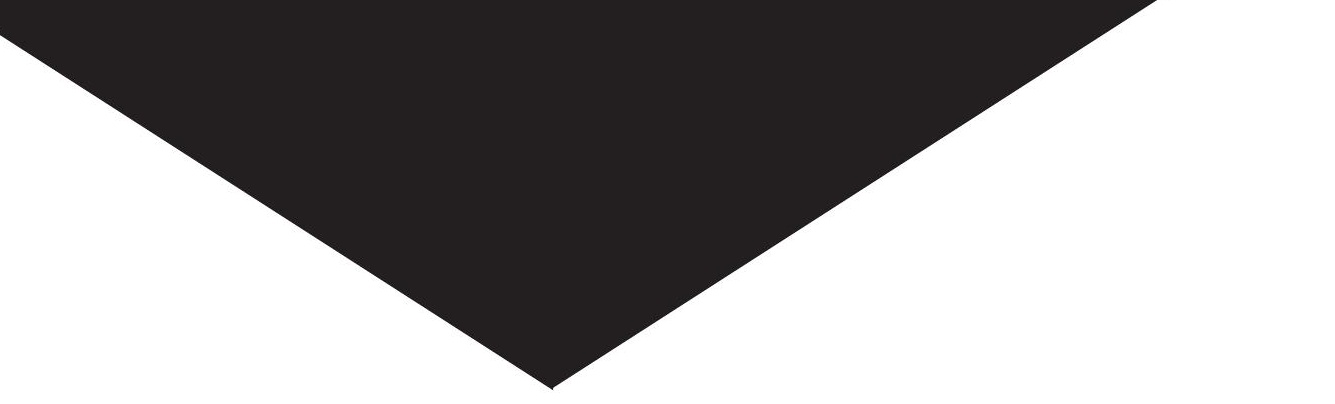

Whis translation is intended for both the scholar and the general 1 reader with an interest in classical Korean fiction. It provides an approachable translation, detailed explanations of culturally specific terms, and in-depth commentary on obscure sections to further scholarly discussion.

The translation is based on the oldest extant woodblock edition from Wansan (wanp'an), which has IO4 leaves in three volumes (33-33-38), the first of which dates from the chongsa 丁巳 year (I857). ${ }^{\mathrm{I}}$ It also draws upon invaluable modern collated editions, such as those by Yi Hŏnhong (Seoul: Koryŏ taehakkyo minjok munhwa yŏn'guso, 1996) and Cho Hŭiung (Seoul: Chimanji, 2009), whose annotations and interpretations are based on IO4-leaf Wansan editions. ${ }^{2}$ In addition, I consulted as many other commercial editions as possible to fill in gaps, correct errors, and ensure a more accurate and comprehensive translation. Examples of other editions I consulted include the following: 
I. 97 leaves in three volumes (33-33-3I), Wansan sin'gan 完山新刊, the imjin year (I892); at Sogang University

2. 89 leaves (30-30-29), n.p., n.d.; at Ewha Womans University, and also University of Tokyo (Ogura Collection)

3. 8o leaves (30-26-24), Wannam sin'gan 完南新刊, the musul year (I898); at C. V. Starr East Asian Library, University of California at Berkeley

4. 8o leaves, manuscript, Posŏng, Chŏlla-namdo, the imja year (I9I2?); at University of Tokyo (Ogura Collection)

This translation includes new research on available editions of the Tale of Cho Ung, and also on the references to classical texts found in the tale. As a result, it corrects some errors of earlier interpretations. The present volume also supplies a more elaborate context for the Tale of Cho Ung and a variety of alternate interpretations, inviting a detailed appreciation of the story.

Searching for a good translation model in modern Korean, I also consulted recent versions of the Tale of Cho Ung. Most of these are educational rewritings based on the short Seoul editions; the literary and cultural value and deep emotional world of the Wansan editions are largely absent. However, Yi Myŏngnang's rewriting of the Tale of Cho Ung in modern Korean (Ch'angbi, 2005), though abbreviated, reveals the core value and literary emotion of the original story, which is one of my aims in producing a translation that will appeal to modern readers.

In translating the Tale of Cho Ung into English, my first challenges were how to treat the traces of oral storytelling-for example, repetitions and the use of formulaic and idiomatic expressions - and how to interpret unidentified or abstruse terms and phrases. The complicated nature of the text makes a literal translation almost impossible. Crafting a reader-friendly translation that conveys the mood and savor of the original text requires delicate and careful judgment. To make this popular tale available to modern readers, I therefore set rules for translation.

First, this translation seeks to convey all the meanings expressed in the original text through a combination of direct and paraphrastic 
interpretations, applied to varying degrees on a case-by-case basis. Fixed terms and clear sentences are translated word for word, while other passages are paraphrased in order to communicate the full meaning of the text to modern readers. Likewise, the translated songs and poems do not always have the same numbers of lines as the originals.

Second, whereas the original text tends to use the same term or expression throughout to describe a similar or recurring situation, I have varied the corresponding English translation slightly depending on the specific context, particularly when translating honorific titles such as kongja and idioms or allusions such as paekkollanmang and namga ilmong. Exact repetition is not always helpful.

Third, the translation does not retain habitual repetitions from the original text when they are unnecessary or hinder the readability of the tale. This is especially true of juxtapositions of the Korean reading of Sino-Korean words and the explanation of those words within a sentence. However, when the original text narrates a Sino-Korean poem and then provides an explanation in Korean to instruct the audience, I include both cases so as to preserve the storytelling environment in which the tale is rooted.

Fourth, the translation uses brackets [] for any addition that is not part of the original text (e.g., for restored phrases from other editions or to supply a word the reader needs to know). Brackets also enclose paraphrased expressions that elucidate unidentified names, places, and times or that summarize earlier events (such as mowŏl moil, irŏ kurŏ, iri iri, and yöch'a yŏch'a). When necessary, I elaborate on such expressions to help readers understand the context.

Finally, this translation uses the McCune-Reischauer romanization system and the Chinese pinyin system. Though the tale is set in China, the Korean reading is applied to names and places in the original text, so there are many places whose Chinese characters are not confirmed. In the notes, however, I use Korean, Chinese, or both, and include original Chinese characters for names and places whenever they could be readily identified or were already available in other manuscript editions. For modern Chinese place names, I use the Chinese reading, along with simplified Chinese characters. In translating official 
titles, I used both Chinese and Korean sources, including Charles Hucker's A Dictionary of Official Titles in Imperial China (Stanford: Stanford University Press, 1985) and the Glossary of Korean Studies by the Academy of Korean Studies.

These conventions will allow students and scholars of East Asian studies to engage more deeply with the textual characteristics of the original tale, discovering differences and commonalities between the Chinese and Korean languages and cultures. I hope that my efforts to create a readable translation that addresses the complicated nature of this story will enhance our understanding of premodern Korean and East Asian literature and allow a new generation of readers to enjoy the exciting Tale of Cho Ung. 
THE TALE OF

CHO UNG 
